\title{
State of the Art and Future Developments for Surgical Planning in LDLT
}

\author{
Cristiano Quintini • Federico Aucejo • Koji Hashimoto • \\ Nizar Zein - Charles Miller
}

Published online: 9 January 2014

(C) Springer International Publishing AG 2014

\begin{abstract}
Living donor liver transplantation (LDLT) is a wellestablished strategy to decrease transplant waiting list mortality. The challenges facing the field relate primarily to donor risk and recipient graft function. The transplant community has developed medical and surgical innovations to address these challenges. This article describes the state of the art and future directions for LDLT surgical planning.
\end{abstract}

Keywords Living donor liver transplantation (LDLT) . Surgical planning $\cdot 3 \mathrm{D}$ model $\cdot 3 \mathrm{D}$ organ printing $\cdot$ Liver volumetry $\cdot$ Surgical navigation

\section{Introduction}

Living donor liver transplantation (LDLT) is a wellestablished strategy to decrease liver transplant waiting list mortality. While LDLT is routinely used in pediatric programs, routine adult-to-adult LDLT is confined to select centers around the world. The complexities of the procedure, along with the donor risks, are the biggest obstacles to widespread use of this valuable treatment option. Preparation for surgery is the single most important determinant of successful outcomes for both donors and recipients. For that reason, all LDLT preoperative studies are designed to provide the most accurate information about anatomy, volume and function of the graft and remnant donor liver. These data are integrated with recipient clinical information to determine the optimal surgical strategy. This manuscript describes the current state of the art and future directions of LDLT surgical planning.

C. Quintini $(\bowtie) \cdot$ F. Aucejo $\cdot$ K. Hashimoto $\cdot$ N. Zein $\cdot$ C. Miller Liver Transplant Program, Digestive Disease Institute - Cleveland Clinic, 9500 Euclid Avenue, Cleveland, OH, USA

e-mail: quintic@ccf.org

\section{Donor Assessment}

Radiological and 3D Modeling

\section{Radiological Imaging and Liver Volume}

The assessment of a potential living donor begins with imaging studies to determine donor liver volume as well as the vascular and biliary anatomy of the liver. The donor patient is placed on a standard multi-detector CT scanner and a 1-2 mm incremental reconstruction is performed in both the arterial and venous phases. In our Institution, these images are sent to MEVIS distant service (Bremen, Germany) by an encrypted web transfer protocol for volumetric and anatomic analysis using proprietary FDA-approved software. This technology and the resulting information have been used successfully in the surgical planning of more than 4,500 LDLTs worldwide [1].

Total and segmental liver volumes are calculated using software-assisted image post-processing (SAIP) which has been proven to be the most accurate method for calculating these volumes, with a reported calculated versus actual volume difference of approximately $17.5 \mathrm{ml}$ and a percentage of error of approximately $2.8 \%[1,2]$. Since the software calculates the liver volume, which is a combination of liver parenchyma and blood filling the sinusoids, a correction factor of 0.9 is needed to predict the actual liver weight after procurement [3, 4]. Right and left graft volumes/weights are obtained therefore according to the desired transection line and inclusion/exclusion of the middle hepatic vein (Fig. 1). Left lateral segment and subsegmental volumes are also calculated when pediatric transplants are contemplated.

\section{D Modeling and Vasculature}

Using a semi-automated process, the intrahepatic and extrahepatic vasculature is reconstructed three-dimensionally to obtain portal, arterial and hepatic venous territories. Vascular 


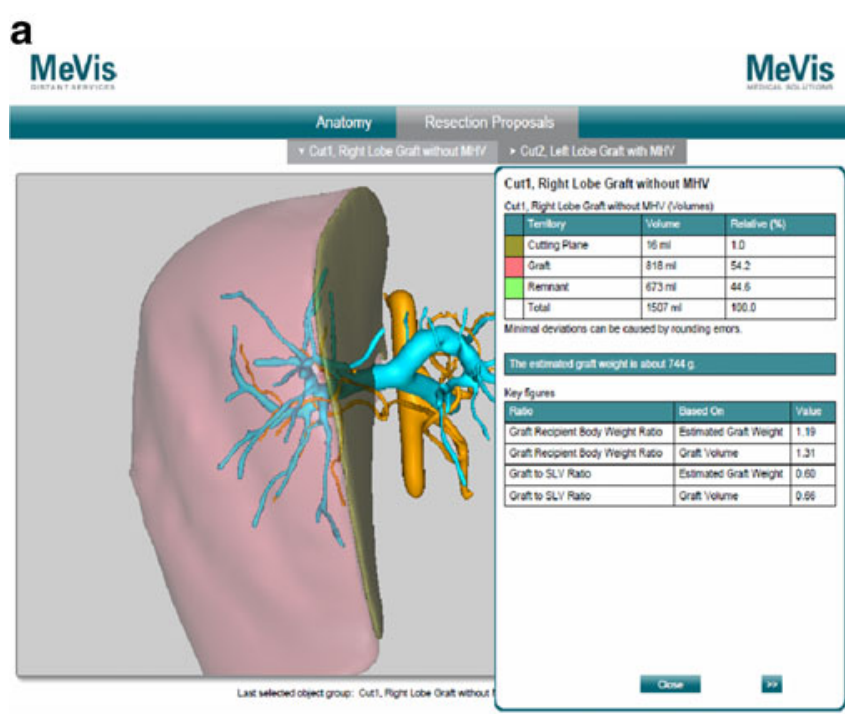

\section{b}

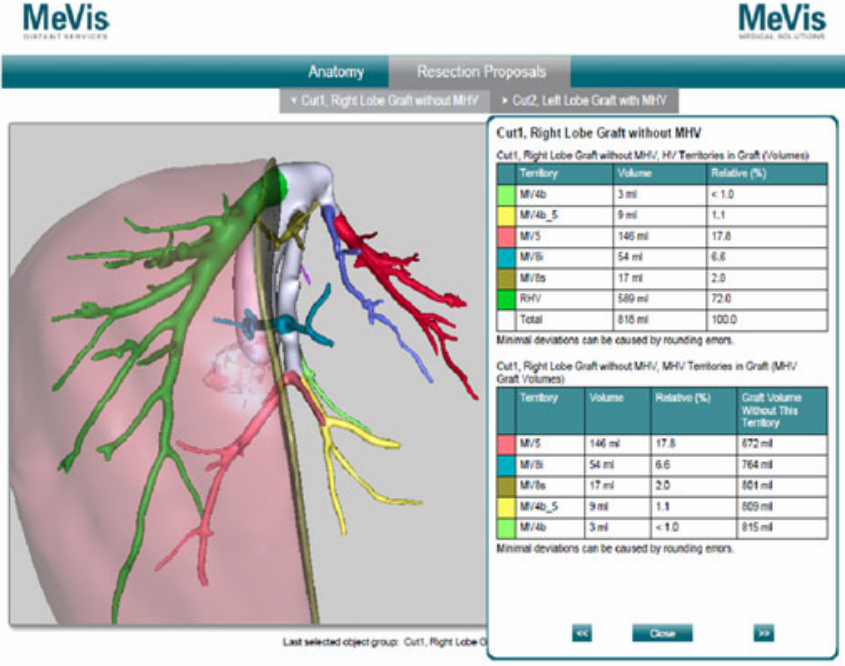

\section{C}

d
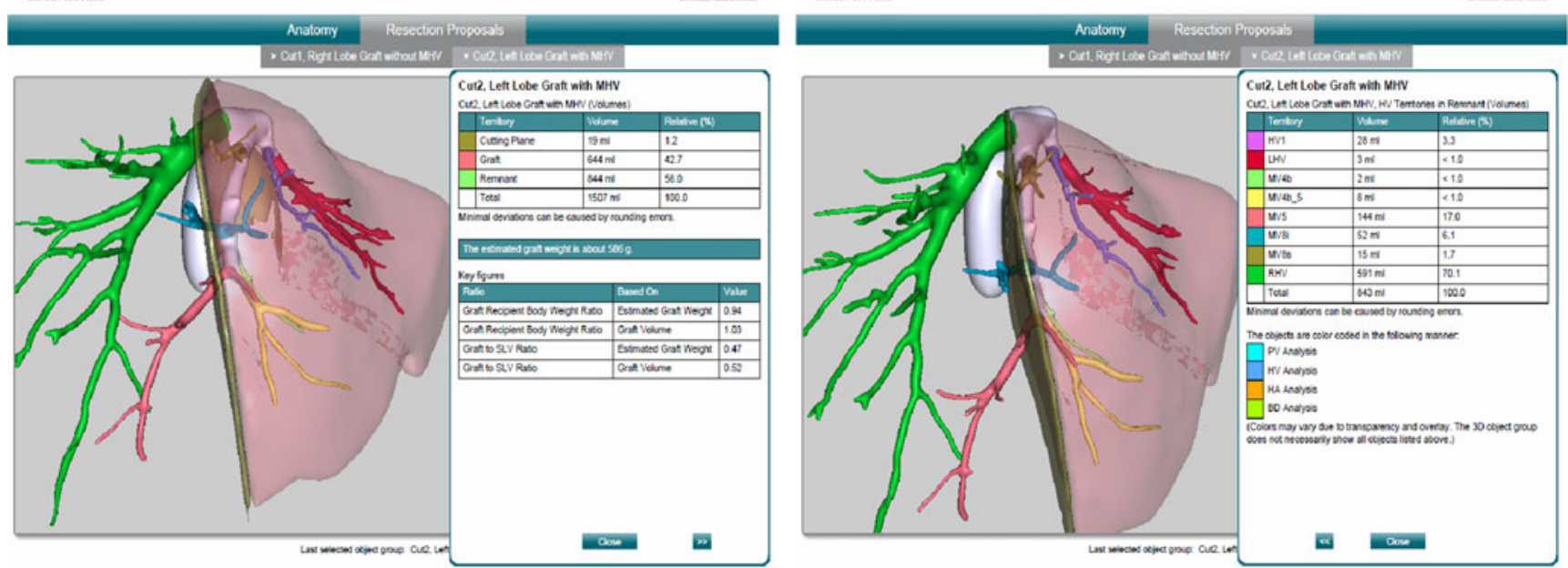

Fig. 1 Right and left graft volumes/weights estimation according to the desired transection line

structures and the corresponding territories are color coded to provide a visual and quantitative (volume) representation of liver segments (Fig. 2).

Three-dimensional (3D) reconstruction of hepatic and portal veins has been shown to be better than, or equivalent to, angiograms [5] in delineating anatomy. It also offers the advantage of having all the vascular structures represented in one 3D image. Hepatic venous 3D reconstruction identifies the exact outflow anatomy of the liver, the spatial relationship of main and segmental veins (important when considering different surgical reconstruction techniques) and more importantly, provides the surgeon with a precise estimation of the liver territories that would undergo venous congestion if the correspondent venous outflow was sacrificed. Such estimation is very important since outflow compromise is associated with impaired regeneration, biliary complications, and potentially small for size syndrome [6-8].
Assessment of venous congestion is crucial when considering right lobe grafts, the most commonly used graft in adult to adult LDLT. Right lobe grafts, in fact, share the venous drainage of the anterior segments (5 and 8) with the left lobe (segments $4 \mathrm{a}$ and $4 \mathrm{~b}$ ) by means of the middle hepatic vein, which can be either retained or excluded from the graft. Our preferred approach is to exclude the middle hepatic vein from the graft because it is safer for the donor. This approach, combined with 3D reconstruction and estimation of segment 5 and segment 8 volumes, allows for a precise estimate of the graft recipient body weight ratio (GRBW) and helps guide venous reconstruction on the recipient (for instance, when inferior segment 6 veins are present) (Fig. 3). The experience of some transplant groups provides evidence that the use of 3D modeling [9-11] can be a crucial tool when making surgical determination to involve the middle hepatic vein in right lobe grafts. 
Fig. 2 Hepatic venous territories. Vascular structures and the corresponding territories are color coded to provide a visual and quantitative (volume) representation of liver segments

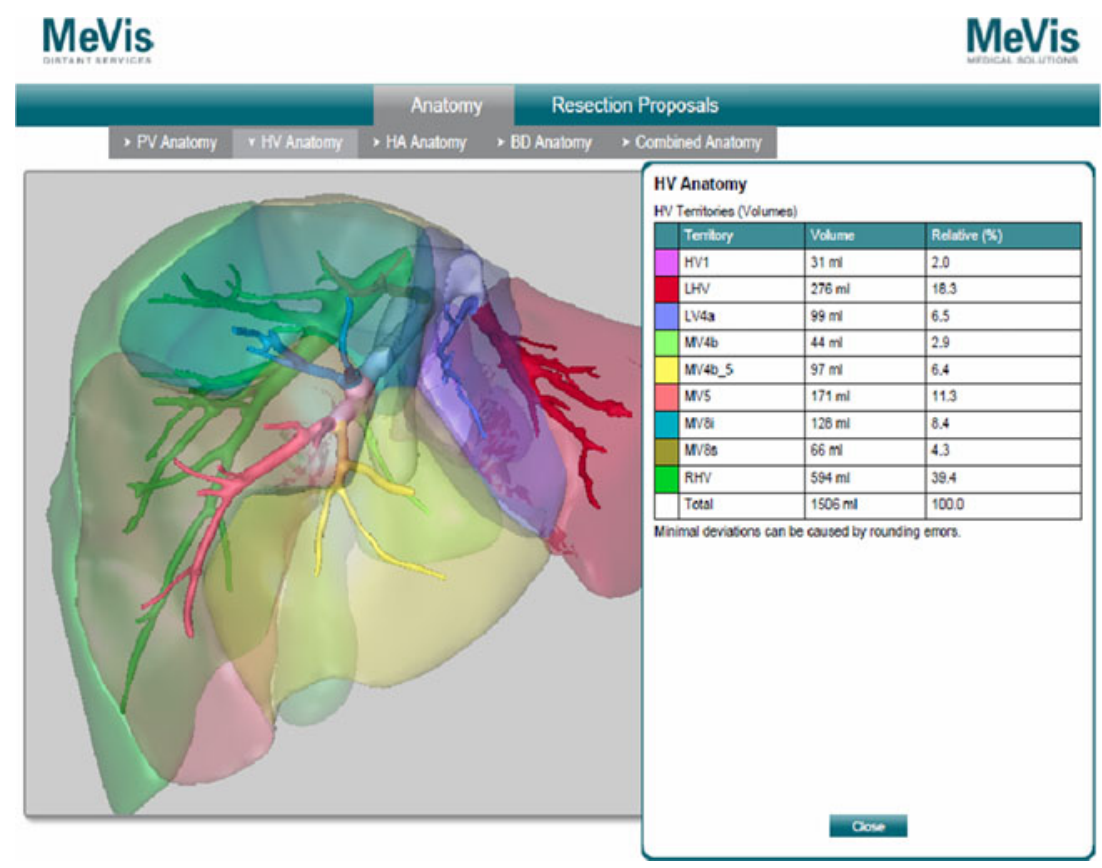

\section{Preoperative Assessment of Biliary Anatomy}

Preoperative assessment of the bile duct anatomy is important for surgeons in selecting the appropriate donor and in planning the surgical approach. A variety of anomalous branching patterns can affect the surgical approach and even preclude liver donation [12, 13]. Magnetic resonance cholangiopancreatography (MRCP) is most commonly used for preoperative assessment of biliary anatomy. MRCP allows visualization of bile duct branching patterns in the majority of cases. Computed tomography (CT) cholangiography, with the use of intravenous contrast, provides a visual of the intrahepatic biliary tree in those cases where the MRI does not provide enough anatomical details [12]. CT cholangiopathy, however, requires additional radiation. Additionally, the contrast agent has a well-documented risk of allergic reaction [14]. Similarly, endoscopic retrograde cholangiopancreatography (ERCP) is a relatively invasive procedure with a potential risk of procedure-related complications. ERCP has been abandoned by most programs, including ours [15].

At our institution, contrast-enhanced multi-phasic CT angiography is used to assess vascular anatomy on all potential LDLT donors. T2-weighted MRCP is used to evaluate the biliary anatomy. All donors undergo intraoperative cholangiogram for anatomy confirmation and determination of the duct transection line. Once the MRCP is obtained, the images are fused with those obtained with the CT scan. Threedimensional modeling allows the integration of all the relevant anatomical structures (portal vein, hepatic artery and bile duct) in one image along with the virtual parenchymal transaction line. The gallbladder (and other anatomical structures) can also be included in the model and used as a landmark for the establishment of the transection line. The interactive manipulation of this image prior to surgery helps simulate the changes in perspective experienced during the actual operation (mobilization and rotation of the liver), thus allowing the surgeon to better understand spatial relationships among critical structures during the different phases of the operation. Importantly, this serves to minimize intraoperative complications [16].

\section{Using 3D, Solid Object Organ Printing for Preoperative} Planning in Live Donor Liver Transplantation

While 3D reconstruction is uniformly used in the living donor field, it presents several drawbacks, the most important one being the representation of $3 \mathrm{D}$ images on a $2 \mathrm{D}$ computer screen. Three-dimensional (3D) printing is a process of making a 3D solid object from a digital model. This method creates real objects from images, resulting in a tangible indication of depth. The ability to easily and repetitively rotate the actual model prior to, and during, surgical operations gives the surgeon greater ability for intuitive navigation of critical anatomical areas compared with navigation ability using static monitor representation. Furthermore, the printed 3D liver offers the opportunity to simulate real life intraoperative manipulation (e.g., mobilization and retraction of the liver, exposure of the hepatic hilum, etc.) allowing for easy re-orientation and identification of anatomical landmarks. Another key aspect of the printed liver is the transparency of material used for printing, along with the use of specific color codes for vascular and biliary structures.

Our group recently developed a prototype of a human liver using 3D printing technology based on a patient's individual CT scan and magnetic resonance imaging (MRI) [17••] (Fig. 4). This model was prospectively studied on three 
Fig. 3 Three-dimensional reconstruction and estimation of segment 5 and segment 8 volumes. An Inferior hepatic vein is also depicted draining segment 6

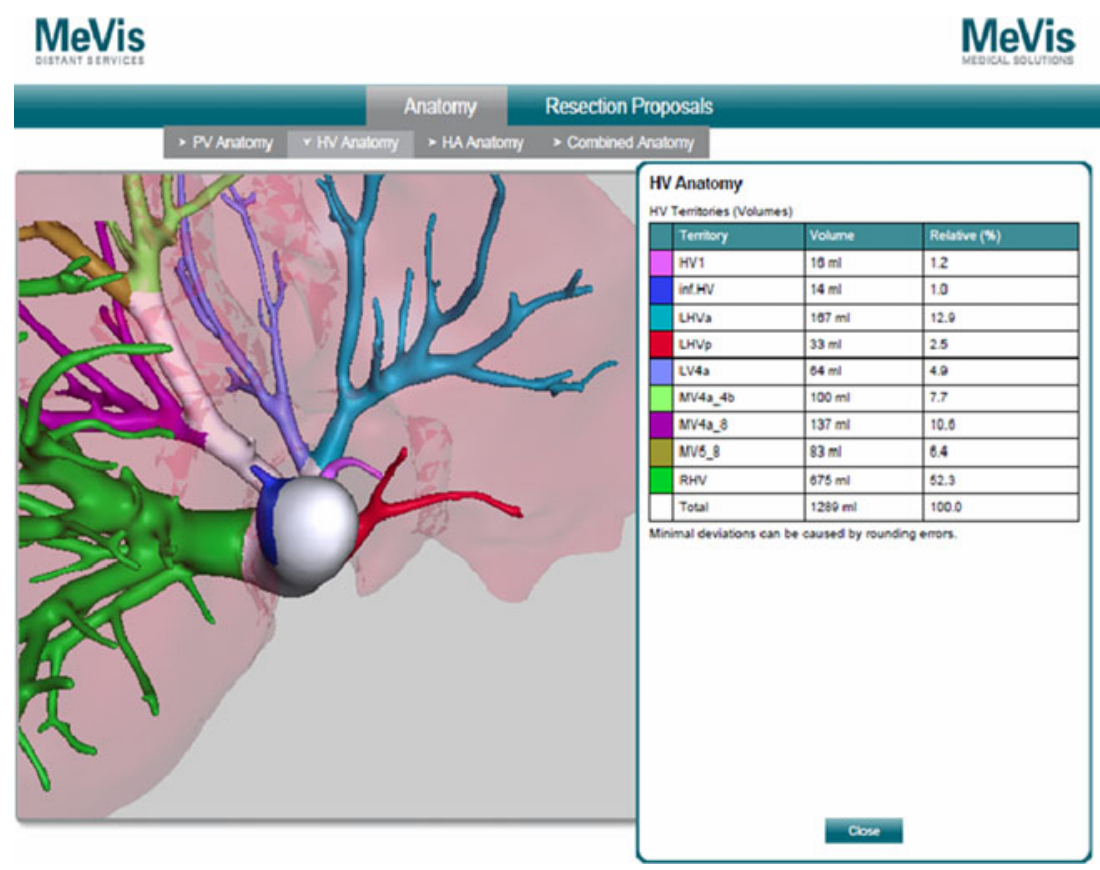

consecutive patient pairs (three donors and three recipients) who were evaluated and approved for LDLT at our Institution. Through direct comparative validation protocol, these models were shown to have very high accuracy, with a mean dimensional error of less than $4 \mathrm{~mm}$ for the entire model and less than $1.3 \mathrm{~mm}$ for vascular diameter (portal vein and its main branches, hepatic veins or hepatic artery) (Fig. 5).

Our preliminary experience shows that 3D organ printing can be a valuable tool in understanding spatial relationships of vascular and biliary anatomical structures, ultimately facilitating surgery and potentially minimizing intraoperative complications. These highly accurate models provided practical and hands-on tools with a number of possible unique applications for surgical planning and medical education. Future refinement of 3D printing and the modeling process may further complement and

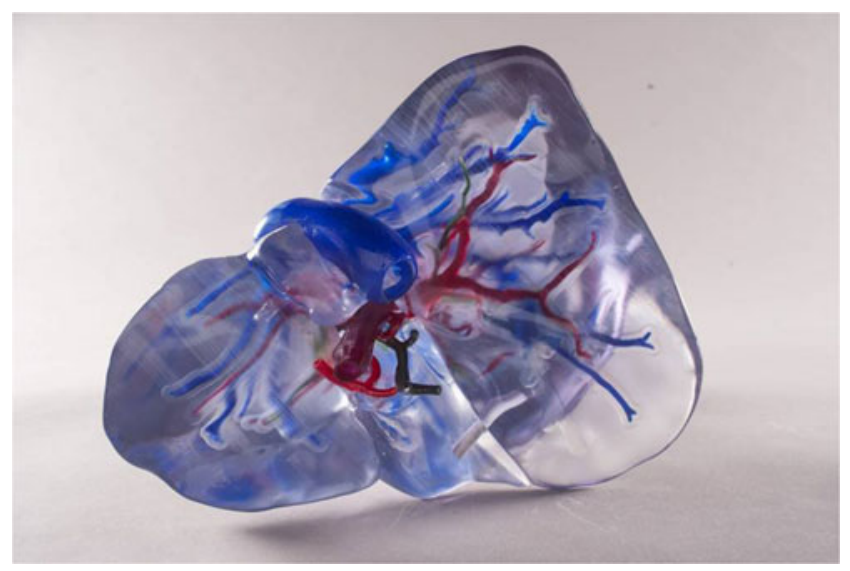

Fig. 4 Prototype of a human liver using 3D printing technology based on a patient's individual CT scan potentially replace the information provided by intraoperative studies (Doppler ultrasound and intraoperative cholangiogram).

\section{Recipient Preoperative Assessment and Planning}

Successful LDLT relies on both graft (quality, actual and functional size) and recipient factors. Therefore both donor and recipient aspects are taken into consideration when deciding what type of graft to procure (right vs left).

On the donor side, the magnitude of the hepatectomy correlates with specific morbidity and mortality rates, with the right hepatectomy operation carrying the highest risk [18].

On the recipient side, the volume of the donor graft must be able to tolerate the increased portal flow and pressure that characterizes cirrhotic patients in order to avoid the small for size syndrome (SFSS).

SFSS is a clinicopathological situation occurring after graft reperfusion in which excessive portal inflow both damages the sinusoidal vascular bed inducing hepatocyte ischemia and affecting graft function as manifested by cholestasis, coagulopathy and ascites [19].

\section{Inflow Modification}

In general, for a LDLT to be successful, the graft size should be as large as $30-40 \%$ of the standard liver volume for the ecipient or about $0.8 \%$ of the recipient body weight. In terms of portal vein flow, this should be less than $300 \mathrm{ml} / \mathrm{min} / 100 \mathrm{~g}$ of liver tissue [20]. In order to both minimize donor risk and maximize recipient graft function, the use of the left lobe (safer for the donor) and the use of inflow modification (IM) measures to prevent excessive portal vein flow (in the recipient) have been 
Fig. 5 Three-dimensional models were shown to have very high accuracy

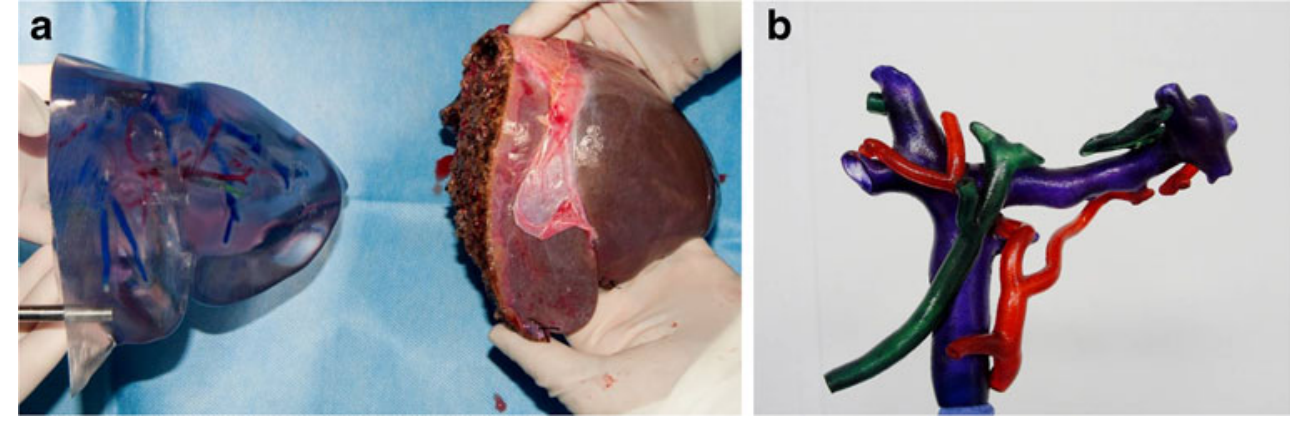

implemented. These graft inflow modulations include splenic artery ligation, splenectomy, or hemiportocaval shunt [21•,22]. While specific guidelines are lacking, experience based on preoperative and intraoperative measurement of hemodynamic parameters can guide decisions as to when IM is indicated.

Preoperative assessment of the recipient's hemodynamics is essential in determining if a larger or smaller graft volume should be utilized. For instance, a portal pressure $\leq 15 \mathrm{mmHg}$ has been associated with improved survival and in this situation a graft recipient weight ratio below $0.8 \%$ could be considered safely [23]. In line with this, it is the standard practice of our group to assess the following recipient hemodynamic parameters preoperatively: right atrial pressure, free hepatic venous pressure, wedged hepatic venous pressure, hepatic vein pressure gradient (hepatic wedged pressure minus hepatic free pressure), right ventricular pressure, pulmonary artery pressure, pulmonary wedged pressure, cardiac output and cardiac index.

In addition, preoperative radiologic assessment of portal hypertension via triphasic $\mathrm{CT}$ of the abdomen is very relevant. Elements to evaluate include level of splenomegaly, size of splenic artery, ascites and presence of large spleno-renal shunt or other porto-systemic shunts. For instance, patients with decompressed portal vein pressure due to the presence of large spontaneous spleno-renal shunts could be considered optimal candidates for smaller grafts such as left lobes [24].

Intraoperative assessment of portal hypertension via clinical judgment and direct measurement is critical to determine the need and type of IM measures. It is our practice, as well as that of other transplant centers, to measure portal vein pressure via a catheter inserted in the inferior mesenteric vein. The hepatic vein pressure gradient is calculated using the difference between the portal vein pressure and the central venous pressure. These measurements are taken before the hepatectomy and after graft implantation. In our experience, observing a transhepatic gradient of $\geq 10-15 \mathrm{mmHg}$ after the graft has been implanted would indicate the need for IM.

Portal vein and hepatic artery flow measurement and assessment of the hepatic artery buffer response are also critical to determine the need for IM. A portal vein flow greater than $250-300 \mathrm{ml} / \mathrm{min} / 100 \mathrm{~g}$ of liver tissue would be considered detrimental and therefore IM should be performed. If hepatic artery flow is under $100 \mathrm{ml} / \mathrm{min}$ and is associated with good hepatic artery buffer response (increased hepatic artery flow after temporary clamping of the portal vein), IM should also be pursued. Another indication for IM has been the absence of hepatic artery diastolic flow that improves after temporary clamping of the portal vein.

In our practice the magnitude of IM is based on correction of the portal vein pressure and flow after IM was implemented. Splenic artery ligation, splenectomy and hemiportocaval shunt are performed in that order if needed to correct both portal vein and hepatic artery hemodynamics.

\section{Outcomes of Small Grafts with the Utilization of Inflow Modification}

In order to minimize the risk of the donor, efforts have been made to enhance the utilization of left lobe grafts. However, the caveat associated is the increasing possibility of inducing small for size syndrome (SFSS) shifting the risk to the recipient [21 •].

Despite the fact it is well established, the risk of SFSS is increased when the graft weight/recipient weight (GW/RW) ratio is $<0.8 \%$ or the graft weight/standard liver volume (GW/SLV) ratio is $<40 \%$; various reports support the use of smaller grafts with excellent patient and graft survival. These reports sustain the cause of SFSS is multifactorial including the size of the donor graft, degree of portal hypertension and general condition of the recipient manifested by the MELD score; suggesting, therefore, a key component of successful outcome requires careful recipient selection and implementation of IM if needed [25-28].

Soejima et al. [27] compared the outcome of living donor liver transplantation using left and right lobe grafts. The authors concluded that left lobe grafts should be considered as much as possible for donor safety; however, they should not be used in recipients with MELD score $>30$ if GW/SVL is $<35 \%$. Additionally the implementation of splenectomy alone or in combination with hemiportocaval shunt was critical when using extra-small grafts. In line with this, Hill et al. [29] compared outcomes between recipients undergoing transplantation with GW/RW ratio $<0.8 \%$ (average MELD 15.7 ) and $\geq 0.8 \%$ (average MELD 17.7) with similar results. In another study, Troisi et al. [26] compared recipients receiving small grafts with 
GW/RW ratio $\leq 0.8 \%$ with and without IM via hemiportocaval shunt. The utilization of IM improved 1 year patient and graft survival from $40 \%$ to $87.5 \%$ and from $20 \%$ to $75 \%$, respectively. Botha et al. [28] reported 21 left lobe adult LDLTs with IM via hemiportocaval shunt in 16 patients with a median GW/RW ratio of $0.67 \%$ reducing the median portocaval gradient from 18 to $5 \mathrm{mmHg}$. Ogura et al. [23] reported the use of splenectomy alone or in combination with meso-renal shunt to keep the portal pressure after reperfusion under $15 \mathrm{mmHg}$. This increased the utilization of small grafts $(\mathrm{GW} / \mathrm{RW}<0.8 \%$ ) from 7.8 to $23.9 \%$ and the utilization of left lobes from 4.9 to $32.1 \%$.

Despite the evidence of the advantage of IM in optimizing the utilization of small grafts and specifically left lobe grafts, there are other factors - as previously mentioned - that contribute to SFSS in addition to portal hyper perfusion/pressure. An example of this is reflected in the study by Ishizaki et al. [30] where in 24 of 42 left lobe adult LDLTs, the GW/SVL was $<40 \%$ and in 17 cases the GW/RW ratio was $<0.8 \%$. The mean portal pressure after reperfusion was $21.5 \pm 3.6 \mathrm{mmHg}$ and no IM was implemented. There were no SFSS and patient and graft survivals were 100, 97 and $91 \%$ at 1, 3 and 5 years respectively.

Additionally, since usually the volume provided by a left lobe graft is $<40 \%$ of the total liver volume, efforts to include the caudate lobe with the left graft should be considered since this can increase the graft volume up to $9 \%$ in most cases [31].

In summary, when selecting small grafts balancing the risk between the donor and recipient should consider both donor and recipient variables. These should include the graft size, donor age, recipient overall condition including MELD score and level of portal hypertension. Under these considerations and with the implementation of IM, it is possible to enhance the utilization of the left lobe improving the donor safety and preserving the recipient's outcome.

\section{Outflow}

Outflow is as important as balanced inflow for the partial graft. A suboptimal outflow would result in a high resistance vascular bed leading to geographic or global graft congestion and ischemia, ascites and potential hepatic artery thrombosis. Reconstruction of segments V and VIII veins in right lobe grafts without the middle hepatic vein as well as large inferior hepatic veins is critical to avoid this complication. Similarly, enhancement of the ostium of the graft's main hepatic vein via venotomy and venoplasty with a patch of cryopreserved or synthetic vascular graft is recommended and standard practice of our group as well as others [32, 33]. The indication to reconstruct segments $\mathrm{V}$ and VIII or inferior hepatic veins is determined by preoperative assessment via imaging. The magnitude of the calculated liver volume drained by these veins related to the entire graft volume will guide this determination. Ultimately, intraoperative assessment of the size of these veins defines the need for reconstruction [34]. Based on the very complex hemodynamics that partial grafts are subjected to, a critical part of the operation is to avoid technical error when reconstructing the hepatic artery. Our group approaches the hepatic artery reconstruction under a microscope for most of the cases.

In summary, a complex relationship between hemodynamic, anatomical and physiologic variables challenges the practice of LDLT. Preoperative assessment via imaging technology, direct hemodynamic monitoring and clinical judgment are current tools to aid decision making in determining type of graft to balance the risk between the donor and the recipient. Continued assessment of these variables intraoperatively via direct pressure and flow measurement is essential to define the need and type of inflow modification to enhance graft function and or prevent small for size syndrome.

Future Directions: Computer-Assisted Surgical Navigation for Living Donor Liver Transplant

Computer-assisted surgery has been recognized as the standard of care for surgical procedures in numerous fields such as neurosurgery, orthopedics, and ENT [35, 36]. Only recently has this technology been introduced to surgery involving softtissue abdominal organs such as the liver [37]. A primary advantage of this technology is that tracked surgical instruments provide real-time visual information of the surgical field and the anatomical structures previously shown only in preoperative imaging studies (CT and MRI). This technology works through an optically tracked adapter attached to the surgical instrument (CUSA dissector, Bovie, ultrasound probe, etc.). Among other capabilities, the tracked instruments improve accuracy in intraoperative navigation by identifying anatomical landmarks (falciform ligament and inferior edges of the liver, right and left triangular ligaments). A portable dual-monitor computer station positioned next to the operating table allows for real-time visualization of up to two optically tracked devices. Tracked

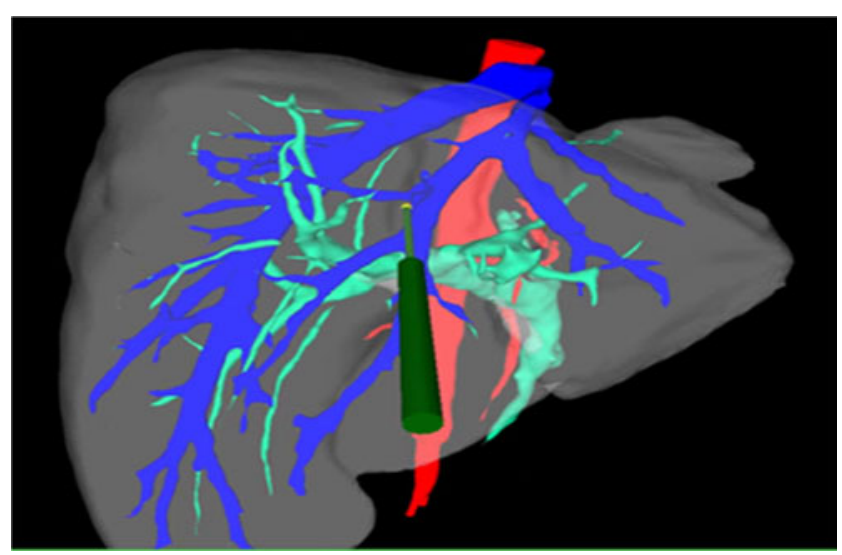

Fig. 6 Tracked instrument position is represented real time in a computer screen next to the operating table. Hepatic vasculature is depicted in transparency. The CUSA dissector is the tracked device in this picture, but any instrument can be tracked 
instrument position is overlaid on the axial, sagittal, coronal and oblique views of the preoperative image (Fig. 6). This technology improves the surgeon's spatial understanding of the vital structures that will be encountered during surgery and minimizes the risks associated with the donor hepatectomy. While the traditional preoperative radiologic imaging has allowed the surgeon to build a mental roadmap prior to the operation, the introduction of computer-assisted navigation allows for the transfer of this vital information directly to the surgical field in real-time, where it can be consulted regularly during the operation to confirm adherence to the surgical plan. The accuracy of this device once anatomical landmarks are registered is in the order of 2.5-3 $\mathrm{mm} \mathrm{[38].}$

We have used this system in cases of living donor hepatectomies, primarily to determine feasibility and to validate our assessment of the device (Pathfinder Therapeutics, Nashville, $\mathrm{TN})$. In our experience, this technology was easy to use, intuitive and helpful in confirming the nature of important anatomical structures encountered during the parenchymal phase of the hepatectomy. For instance, segment 5 and segment 8 hepatic venous branches were accurately identified using the navigation device without need of intraoperative ultrasound (which does not provide a clear image after the transection is in progress due to artifacts from the transection line) and confirmed by direct visualization of the veins. One technical limitation of the device is the inability to continuously monitor organ movement and deformation. This is mitigated in part by the relatively quick landmark registration process, which is required every time the liver is moved from a steady position.

In our opinion, tracked surgical devices hold a great potential in living donor liver hepatectomies where integration of preoperative studies in real-time surgery could improve accuracy and decrease complications. For a complete adoption of this technology into clinical practice, it would be important to improve accuracy during the manipulation of the organ as well as to eliminate the need for continuous landmark registration.

Cost-benefit Considerations for the use of Advanced

Technologies

When considering the introduction of new technologies to the field of medicine usually that comes with a twofold hurdle. One important aspect has to do with the expense associated to these advanced technologies in an era of increasing population and rising healthcare cost; while the other relevant part of it has to do with the validation of its effectiveness when compared with standard methods.

In this chapter we mentioned the utilization of 3D modeling software, 3D printing and image guidance surgical navigation. While some of these technologies have been incorporated into our standard LDLT practice, such as 3D modeling software, others are still maturing. At the time of this publication, the cost of performing 3D image modeling is in the range of US\$ 800
1,600 , depending on the complexity of the reconstruction and information incorporated in the analysis. The production of 3D printed models is still at a development phase with costs (including labor and material) around US\$ 3,000-4,000. This cost is projected to decrease dramatically as we become more familiar with the process. Finally, the cost for the acquisition of the equipment to perform image-guided surgical navigation is approximately US\$ $100,000-200,000$ with a cost for disposable of about US\$ 200-300 per case.

Most of these technologies in their current form are meant to add quality and complement but not replace standard methods such as ultrasound, CT and MRI. Therefore, the benefit to the patient - that is, the value added by these technologies to the practice of LDTL-will have to be subjected to the analysis of cumulative surgical and clinical experience.

\section{Conclusions}

The ultimate goal of professionals involved in the challenging practice of LDLT is to continuously improve donor safety while maximizing recipient outcomes. Technological advancements established and initiated within the transplant community have gone a long way toward fulfilling this goal. Still, living donor liver transplantation is a constantly evolving field. Future innovations, particularly in the field of augmented reality, will continue to make this procedure safer and more effective in the years to come.

\section{Compliance with Ethics Guidelines}

Conflict of Interest Cristiano Quintini, Federico Aucejo, Koji Hashimoto, Nizar Zein, and Charles Miller declare that they have no conflict of interest.

Human and Animal Rights and Informed Consent This article does not contain any studies with human or animal subjects performed by any of the authors.

\section{References}

Papers of particular interest, published recently, have been highlighted as:

- Of importance

-• Of major importance

1. Pomposelli JJ, Tongyoo A, Wald C, Pomfret EA. Variability of standard liver volume estimation versus software-assisted total liver volume measurement. Liver Transpl. 2012;18(9):1083-92.

2. Selle D, Preim B, Schenk A, Peitgen HO. Analysis of vasculature for liver surgical planning. IEEE Trans Med Imaging. 2002;21(11): 1344-57.

3. Satou S, Sugawara Y, Tamura S, Yamashiki N, Kaneko J, Aoki T, et al. Discrepancy between estimated and actual weight of partial 
liver graft from living donors. J Hepato-Biliary-Pancreat Sci. 2011;18(4):586-91.

4. Kim KW, Lee J, Lee H, Jeong WK, Won HJ, Shin YM, et al. Right lobe estimated blood-free weight for living donor liver transplantation: accuracy of automated blood-free CT volumetry-preliminary results. Radiology. 2010;256(2):433-40.

5. Bogetti JD, Herts BR, Sands MJ, Carroll JF, Vogt DP, Henderson JM. Accuracy and utility of 3-dimensional computed tomography in evaluating donors for adult living related liver transplants. Liver Transpl. 2001;7(8):687-92.

6. Lee S, Park K, Hwang S, Lee Y, Choi D, Kim K, et al. Congestion of right liver graft in living donor liver transplantation. Transplantation. 2001;71(6):812-4.

7. Cha CH, Ruo L, Fong Y, Jarnagin WR, Shia J, Blumgart LH, et al. Resection of hepatocellular carcinoma in patients otherwise eligible for transplantation. Ann Surg. 2003;238(3):315-21. discussion 21-3.

8. Akamatsu N, Sugawara Y, Kaneko J, Sano K, Imamura H, Kokudo $\mathrm{N}$, et al. Effects of middle hepatic vein reconstruction on right liver graft regeneration. Transplantation. 2003;76(5):832-7.

9. Asakuma M, Fujimoto Y, Bourquain H, Uryuhara K, Hayashi M, Tanigawa N, et al. Graft selection algorithm based on congestion volume for adult living donor liver transplantation. Am J Transplant. 2007;7(7):1788-96.

10. Radtke A, Sgourakis G, Sotiropoulos GC, Beckebaum S, Molmenti EP, Saner FH, et al. Donor/recipient algorithm for management of the middle hepatic vein in right graft live donor liver transplantation. Am J Surg. 2010;199(5):708-15.

11. Radtke A, Sotiropoulos GC, Sgourakis G, Molmenti EP, Schroeder T, Saner FH, et al. "Anatomical" versus "territorial" belonging of the middle hepatic vein: virtual imaging and clinical repercussions. J Surg Res. 2011;166(1):146-55.

12. Wang ZJ, Yeh BM, Roberts JP, Breiman RS, Qayyum A, Coakley FV. Living donor candidates for right hepatic lobe transplantation: evaluation at CT cholangiography - initial experience. Radiology. 2005;235(3):899-904.

13. Tsang LL, Chen CL, Huang TL, Chen TY, Wang CC, Ou HY, et al. Preoperative imaging evaluation of potential living liver donors: reasons for exclusion from donation in adult living donor liver transplantation. Transplant Proc. 2008;40(8):2460-2.

14. Nilsson U. Adverse reactions to iotroxate at intravenous cholangiography. A prospective clinical investigation and review of the literature. Acta Radiol. 1987;28(5):571-5.

15. Masci E, Toti G, Mariani A, Curioni S, Lomazzi A, Dinelli M, et al. Complications of diagnostic and therapeutic ERCP: a prospective multicenter study. Am J Gastroenterol. 2001;96(2):417-23.

16. Endo I, Shimada H, Sugita M, Fujii Y, Morioka D, Takeda K, et al. Role of three-dimensional imaging in operative planning for hilar cholangiocarcinoma. Surgery. 2007;142(5):666-75.

17.• Zein NN, Hanouneh IA, Bishop PD, Samaan M, Eghtesad B, Quintini C, et al. 3-dimentional (3D) print of liver for preoperative planning in live donor liver transplantation. Liver Transpl. 2013;19(12):1304-10. This article describes the first 3D printed human liver and its application in living donor liver hepatectomy.

18. Florman S, Miller CM. Live donor liver transplantation. Liver Transpl. 2006;12(4):499-510.

19. Kelly DM, Demetris AJ, Fung JJ, Marcos A, Zhu Y, Subbotin V, et al. Porcine partial liver transplantation: a novel model of the "small-for-size" liver graft. Liver Transpl. 2004;10(2):253-63.

20. Sainz-Barriga M, Scudeller L, Costa MG, de Hemptinne B, Troisi RI. Lack of a correlation between portal vein flow and pressure: toward a shared interpretation of hemodynamic stress governing inflow modulation in liver transplantation. Liver Transpl. 2011;17(7):836-48.

21. Roll GR, Parekh JR, Parker WF, Siegler M, Pomfret EA, Ascher NL, et al. Left hepatectomy versus right hepatectomy for living donor liver transplantation: shifting the risk from the donor to the recipient. Liver Transpl. 2013;19(5):472-81. This review summarizes the most relevant aspects inherent to LDLT regarding the surgical risk for both donor and recipient according to the utilization of right lobe vs left lobe grafts.

22. Sánchez-Cabús S, Fondevila C, Calatayud D, Ferrer J, Taurá P, Fuster $\mathrm{J}$, et al. Importance of the temporary portocaval shunt during adult living donor liver transplantation. Liver Transpl. 2013;19(2):174-83.

23. Ogura Y, Hori T, El Moghazy WM, Yoshizawa A, Oike F, Mori A, et al. Portal pressure $<15 \mathrm{~mm} \mathrm{Hg}$ is a key for successful adult living donor liver transplantation utilizing smaller grafts than before. Liver Transpl. 2010;16(6):718-28.

24. Aucejo FN, Hashimoto K, Quintini C, Kelly D, Vogt D, Winans C, et al. Triple-phase computed tomography and intraoperative flow measurements improve the management of portosystemic shunts during liver transplantation. Liver Transpl. 2008;14(1):96-9.

25. Ikegami T, Masuda Y, Ohno Y, Mita A, Kobayashi A, Urata K, et al. Prognosis of adult patients transplanted with liver grafts $<35 \%$ of their standard liver volume. Liver Transpl. 2009;15(11):1622-30.

26. Troisi R, Ricciardi S, Smeets P, Petrovic M, Van Maele G, Colle I, et al. Effects of hemi-portocaval shunts for inflow modulation on the outcome of small-for-size grafts in living donor liver transplantation. Am J Transplant. 2005;5(6):1397-404.

27. Soejima Y, Shirabe K, Taketomi A, Yoshizumi T, Uchiyama H, Ikegami $\mathrm{T}$, et al. Left lobe living donor liver transplantation in adults. Am J Transplant. 2012;12(7):1877-85. In this relevant study experience with the use of left lobe grafts is reported. Surgical strategies such as the use of inflow modification and clinical evaluation for patient selection are thoroughly discussed.

28. Botha JF, Langnas AN, Campos BD, Grant WJ, Freise CE, Ascher NL, et al. Left lobe adult-to-adult living donor liver transplantation: small grafts and hemiportocaval shunts in the prevention of smallfor-size syndrome. Liver Transpl. 2010;16(5):649-57.

29. Hill MJ, Hughes M, Jie T, Cohen M, Lake J, Payne WD, et al. Graft weight/recipient weight ratio: how well does it predict outcome after partial liver transplants? Liver Transpl. 2009;15(9):1056-62.

30. Ishizaki Y, Kawasaki S, Sugo H, Yoshimoto J, Fujiwara N, Imamura H. Left lobe adult-to-adult living donor liver transplantation: should portal inflow modulation be added? Liver Transpl. 2012;18(3):305-14.

31. Raut V, Alikhanov R, Belghiti J, Uemoto S. Review of the surgical approach to prevent small-for-size syndrome in recipients after left lobe adult LDLT. Surg Today. 2013 Aug 1.

32. Hwang S, Ahn CS, Kim KH, Moon DB, Ha TY, Song GW, et al. Standardization of modified right lobe grafts to minimize vascular outflow complications for adult living donor liver transplantation. Transplant Proc. 2012;44(2):457-9.

33. Hwang S, Jung DH, Ha TY, Ahn CS, Moon DB, Kim KH, et al. Usability of ringed polytetrafluoroethylene grafts for middle hepatic vein reconstruction during living donor liver transplantation. Liver Transpl. 2012;18(8):955-65.

34. Yonemura Y, Taketomi A, Soejima Y, Yoshizumi T, Uchiyama H, Gion T, et al. Validity of preoperative volumetric analysis of congestion volume in living donor liver transplantation using three-dimensional computed tomography. Liver Transpl. 2005;11(12):1556-62.

35. Gumprecht HK, Widenka DC, Lumenta CB. Brain Lab VectorVision Neuronavigation System: technology and clinical experiences in 131 cases. Neurosurgery. 1999;44(1):97-104. discussion -5 .

36. Wirtz CR, Albert FK, Schwaderer M, Heuer C, Staubert A, Tronnier VM, et al. The benefit of neuronavigation for neurosurgery analyzed by its impact on glioblastoma surgery. Neurol Res. 2000;22(4):354-60.

37. Widmann G, Schullian P, Haidu M, Bale R. Stereotactic radiofrequency ablation (SRFA) of liver lesions: technique effectiveness, safety, and interoperator performance. Cardiovasc Interv Radiol. 2012;35(3): 570-80.

38. Oldhafer KJ, Stavrou GA, Prause G, Peitgen HO, Lueth TC, Weber S. How to operate a eliver tumor you cannot see. Langenbeck's Arch Surg. 2009;394(3):489-94. 